\title{
Disposition des racines adventives autour de la tige de maïs (Zea mays L.)
}

\author{
S. Pellerin ${ }^{1}$, F. Tricot ${ }^{1}$ et J. Chadœuf ${ }^{2}$ \\ 1 INRA, laboratoire d'agronomie, 68021 Colmar; \\ 2 INRA, laboratoire de biométrie, 84140 Montfavet, France
}

(reçu le 21 janvier 1989; accepté le 30 juin 1989)

Résumé - Nous avons étudié chez le maïs (Zea mays L. variété Déa) la disposition des racines adventives autour de l'axe de la tige. Sur un échantillon de 63 pieds, les positions angulaires des racines ont été notées grâce à un dispositif micrométrique mobile en rotation permettant de repérer dans un plan perpendiculaire à la tige l'angle entre chaque racine et une racine du premier entrenœud choisie arbitrairement comme référence.

Nous avons d'abord étudié la disposition des racines sur chaque entrenœud pris séparément. Un test de $\chi^{2}$ conduit à rejeter l'hypothèse d'une disposition uniformément aléatoire des racines autour de la tige. L'hypothèse d'une disposition symétrique doit être également rejetée. Sous cette hypothèse, les angles entre racines seraient de 360/l, l étant le nombre de racines. Cette hypothèse est vérifiée pour $I=3$, mais elle est contredite pour $I=2$ et $I=4$. Pour $I=2$, les racines ne sont pas opposées mais placées à 120 degrés l'une de l'autre.

Sur 2 entrenœuds successifs, les dispositions sont alternées. Les racines de l'entrencud $E_{i+1}$ occupent les angles entre racines les plus ouverts sur l'entrenœud $E_{i}$. Quand 1 racine d'un entrenœud est comprise entre 2 racines de l'entrenœud précédent, elle occupe une position proche de la bissectrice. La disposition des racines sur un entrencud dépend donc du nombre de racines qu'il porte, de la disposition et du nombre de racines sur l'entrenœud précédent. L'origine de ces dispositions est discutée.

morphologie racinaire - phyllotaxie - tige - maïs

Summary - Arrangement of primary roots around the maize stem. The arrangement of primary roots around the stem was studied on 63 maize plants grown outdoors (Zea mays $L$. F1. hybrid Dea). The angular position of each root around the stem was recorded by measuring the angle between this root and a root of the first internode chosen as a reference.

First, the root arrangement was studied on each internode considered separately. A $\chi^{2}$ test showed that roots were not arranged according to a random pattern (Table III). The assumption of a symmetrical arrangement has to be discarded too. On this assumption angles between roots would be equal to $360 /$, I being the number of roots. This assumption could be accepted for $I=3$ but could not be accepted for $I=2$, or $I=4$. For $I=3$ the modal value of angles between roots was 120 degrees (Fig. 5). In this particular case lots of the root arrangements were close to a symmetrical arrangement (Fig. 4). For $I=2$ very few values were equal to 180 degrees (Fig. 3). For $I=4$ the modal classes of angles between roots were [70-80] degrees and [100-120] degrees (Fig. 6.a).

The relative locations of roots belonging to 2 successive internodes (called $E_{i}$ and $E_{i+1}$ ) was also studied. It appeared that the roots of $E_{i+1}$ occupied the more open angles between the roots of $E_{i}$. This result was shown by studying the locations of the roots of $E_{i+1}$ relative by to the angles between roots of $E_{i}$ classified by descending order. The following ratio: number of $E_{i+1}$ roots between 2 roots of $E_{i} / v a l u e$ of the angle between the 2 roots of $E_{i}$ was constant for angles over 100 degrees but decreased for angles under 100 degrees (Fig. 7). When there was $1 E_{i+1}$ root between $2 E_{i}$ roots, this root was often close to the bisectrix of the angle (Fig. 8). When there were $2 E_{i+1}$ roots between $2 E_{i}$ roots, they tended to separate the whole angle into 3 parts equal (Fig. 9). These results showed that the positions of the roots on successive internodes tend to alternate. Possible origins of such arrangements are discussed. 


\section{INTRODUCTION}

Des travaux récents ont souligné l'importance des caractéristiques géométriques du système racinaire vis-à-vis de son rôle de capteur. Ainsi, les possibilités de prélèvement d'eau dépendent du volume total de sol colonisé et de la disposition spatiale des racines dans ce volume, à échelle centi- à décimétrique (Tardieu, 1987).

Le système racinaire d'une plante est une arborescence constituée d'un ou plusieurs axes porteurs de ramifications. Plusieurs auteurs ont développé des modèles, dits d'architecture, permettant de simuler dans l'espace la mise en place du système racinaire par initiation, allongement et ramification de petits segments (Lungley, 1973; Diggle, 1988; Pages \& Aries, 1988). Ces modèles aboutissent à une représentation tridimensionnelle du système racinaire. On peut en déduire la valeur de variables synthétiques pertinentes par rapport aux possibilités d'absorption: longueur de racine par unité de volume de sol et par horizon (Lv): cartes d'impact horizontales ou verticales et calculs de distance entre racines.

Les données nécessaires à la construction d'un tel modèle concernent le développement, la croissance et la géométrie du système racinaire. Dans le cas d'un enracinement fasciculé, la disposition des racines adventives autour de la tige est un des paramètres nécessaires à une modélisation tridimensionnelle. Sur blé, les racines adventives apparaissent par paires opposées (Klepper et al., 1984). Les résultats obtenus par ces auteurs ont été repris dans plusieurs modèles (Porter et al., 1986; Diggle, 1988). Dans le modèle de Diggle, les racines adventives apparaissent par paire de part et d'autre de la tige. La position autour de la tige des plans contenant les couples successifs de racines est supposée aléatoire. A chaque racine est attribuée une probabilité d'apparition.

Sur maïs, le nombre de racines par entrenœud est variable et augmente avec le numéro de l'entrenœud (Picard at al. 1985). II n'existe aucune donnée relative à leur disposition. L'objectif du travail présenté dans cet article est d'étudier l'arrangement de ces racines autour de l'axe de la tige. Un modèle sera proposé dans un deuxième article.

\section{MATÉRIEL ET MÉTHODE}

\section{Données}

L'arrangement des racines autour de la tige a été étudié sur un échantillon de 63 pieds (variété Dea), cultivés au champ sur sol limoneux profond (argile 20,7\%; limon fin $31,1 \%$; limon grossier $38,2 \%$; sable fin $5,2 \%$; sable grossier 2,9\% - domaine expérimental INRA Colmar). Les techniques culturales ont été celles normalement utilisées en grande culture (labour le 14/11/85 semis le 9/05/86 à 78000 pieds/ha-apport de $140 \mathrm{~kg} / \mathrm{ha}$ de $\mathrm{N}, \mathrm{P}$ et K). Après prélèvement des pieds, les positions angulaires des racines ont été repérées à l'aide du dispositif micrométrique présenté Figure 1. Les racines du pied fixé verticalement étaient visibles au travers d'une plaque transparente mobile en rotation et munie d'un repère. Les positions ont été notées par déplacement du repère sur les racines successives et lecture de l'angle correspondant. Pour chaque pied, une racine de l'entrenœud 2 était arbitrairement choisie comme référence. A partir de cette référence, les positions ont été notées entre 0 et 360 degrés, dans le sens trigonométrique positif, avec une précision estimée à 5 degrés. Chaque racine a été identifiée en fonction du numéro de l'entrenœud porteur (Girardin et al., 1986).

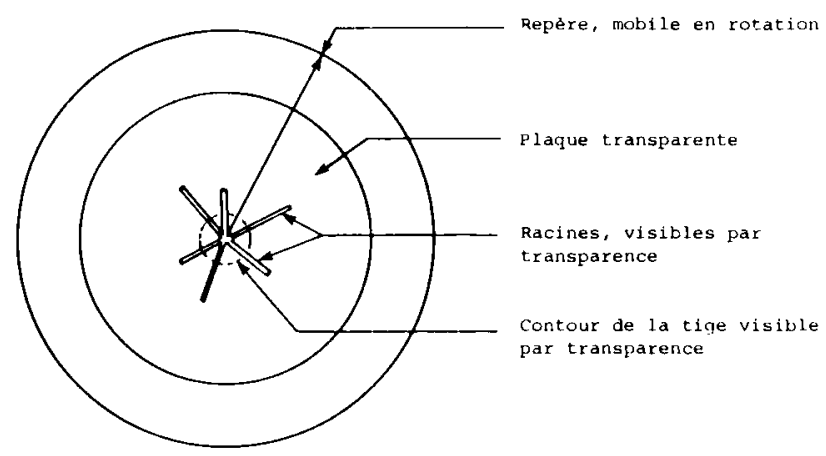

Fig. 1. Représentation schématique du dispositif de mesure, en vue de dessus. La tige est fixée verticalement, avec la base de la tige orientée vers le haut. Les racines sont visibles par transparence et le repère mobile en rotation permet de repérer leur position angulaire.

\section{Méthodes d'analyse}

\section{Disposition des racines sur un entrencud donné}

Nous avons testé 2 hypothèses.

- l'hypothèse d'une disposition uniformément aléatoire et indépendante. Sous cette hypothèse, la probabilité d'apparition d'une racine est uniforme dans l'intervalle [0,360[ et indépendante de la position des autres racines.

- l'hypothèse d'une disposition symétrique. Sous cette hypothèse, si I est le nombre de racines sur l'entrencud, les angles entre racines successives sont égaux à 360/l.

Soit un entrenceud $E_{i}$ portant $I_{i}$ racines notées $R_{j}$ $\left(1<j<l_{i}\right)$. Une racine étant choisie comme référence $\left(R_{1}\right)$, soit $X_{j}$ la variable aléatoire prenant pour valeur la valeur de l'angle entre $R_{j}$ et la référence. Si la position de la racine $\mathrm{R}_{j}$ est aléatoire et indépendante de celle choisie comme référence, la valeur de l'angle entre $\mathbf{R}_{i}$ et la référence résulte d'un tirage équiprobable dans l'intervalle $[0,360[$. La probabilité que cette valeur appartienne à un intervalle donné $x_{j} \pm 180 / n$ est égale à $1 / n$.

$$
p\left(x_{j}-\frac{180}{n}<x_{j}<x_{j}+\frac{180}{n}\right)=\frac{1}{n}
$$


Soit une autre racine $\mathrm{Aj}$; si sa position est aléatoire et indépendante des précédentes, on a

$$
\begin{gathered}
p\left(x_{j},-\frac{180}{n}<x_{j}<x_{j}+\frac{180}{n}\right)=\frac{1}{n} \\
p\left(x_{j}-\frac{180}{n}<x_{j}<x_{j}+\frac{180}{n} ;\right. \\
\left.x_{j} \cdot-\frac{180}{n}<x_{j} \cdot<x_{j} \cdot+\frac{180}{n}\right)=\left(\frac{1}{n}\right)^{2}
\end{gathered}
$$

On peut ensuite généraliser au cas à 1 racires. Pour tester l'hypothèse d'une disposition uniformément aléatoire et indépendante, nous avons confronté par test de $\chi^{2}$ les fréquences observées et calculées dans les intervalles successifs. De façon à avoir des effectifs théoriques par classe suffisants, le calcul a été fait en considérant des intervalles successits de 72 degrés.

Pour décrire les dispositions observées et tester l'hypothèse d'une disposition symétrique, nous avons adapté le mode de représentation des données au nombre de racines. Dans le cas d'un entrencud portant 2 racines et si l'on ne tient pas compte du sens trigonométrique, la disposition est entièrement définie par la valeur d'un des deux angles entre racines. Nous avons décrit ces dispositions par la valeur du plus grand angle. Si la disposition est symétrique, cette valeur doit être proche de 180 degrés. Dans le cas d'un entrenceud portant 3 racines, et si l'on ne tient pas compte du sens trigonométrique, la disposition est entièrement définie par la valeur de deux des trois angles. Nous avons représenté ces dispositions par un point ayant pour coordonnées les valeurs des 2 plus grands angles, notés $\lambda_{1}$ et $\lambda_{2}$. Par construction on a

$$
\lambda_{2}<\lambda_{1}
$$

$\lambda_{2}+\lambda_{1}<360$

$\lambda_{2}>1 / 2\left(360-\lambda_{1}\right)$ (le deuxième angle est supérieur à la moitié de l'angle complémentaire du premier, sinon il ne serait pas le deuxième).

Dans le plan, les 3 droites ainsi définies limitent l'aire des valeurs possibles. Dans le cas d'une disposition symétrique, les points seraient proches du point ayant pour coordonnées $(120,120)$. Au-delà de 3 racines, il n'est pas possible de représenter de manière simple et univoque les dispositions observées. Une disposition est définie par plus de 2 valeurs d'angle et l'ordre dans lesquels ces angles apparaissent autour de la tige a une importance. Dans ce cas, nous avons considéré globalement l'histogramme de toutes les valeurs d'angles entre racines. Si la disposition est symétrique, on doit observer un mode ayant pour valeur $360 / 1$.

\section{Disposition relative des racines sur 2 entre- nœuds successifs}

Soient $E_{i}$ et $E_{i+1} 2$ entrenœuds successifs portant respectivement $I_{i}$ et $l_{i+1}$ racines. Nous avons étudié la disposition des racines de $E_{i+1}$ relativement à $E_{i}$.

Nous avons recherché si les racines de $E_{i+1}$ occupent préférentiellement certains angles entre racines sur $E_{i}$. Nous avons d'abord calculé la fréquence d'occupation d'un angle de $E_{i}$ par 0,1 ou plusieurs racines de $E_{i+1}$ en fonction de la valeur de cet angle. Ensuite, nous avons tenu compte des valeurs relatives des angles entre racines sur $E_{i}$. Pour chaque cas de figure correspondant à un couple de valeurs $\left(l_{i}, l_{i+1}\right)$, nous avons classé les angles entre racines sur $E_{i}$ par ordre décroissant $\left(\lambda_{1}>\ldots>\lambda_{\text {li }}\right)$ et observé dans lesquels de ces angles apparaissent les racines de $E_{i+1}$. Nous avons calculé la fréquence des différentes configurations rencontrées.

Nous avons également étudié la position des racines de $E_{i+1}$ dans les angles entre racines de $E_{i}$ qu'elles occupent. Pour cela, nous avons calculé l'angle entre la (ou les) racine(s) de $E_{i+1}$ et la bissectrice.

\section{RÉSULTATS}

\section{Nombre de racines par entrenœud}

Dans l'échantillon le nombre moyen de racines par entrencud est du même ordre de grandeur que celui habituellement observé (Picard et al., 1985; Jordan et al., 1988) (Tableau I). Sur chaque entrenœud, il existe une variabilité du nombre de racines. Sur 2 entrenœuds successifs, les nombres de racines sont corrélés négativement (Tableau II). Le type de pied le plus fré-

\begin{tabular}{|c|c|c|c|c|c|c|c|c|c|c|c|}
\hline $\begin{array}{l}\text { Nombre de racines } \\
\text { Position de l'entrenœud }\end{array}$ & 0 & 1 & 2 & 3 & 4 & 5 & 6 & 7 & 8 & $\begin{array}{l}\text { Nombre } \\
\text { moyen de } \\
\text { racines }\end{array}$ & Ecart type \\
\hline 2 & & & 3 & 31 & 28 & 1 & & & & 3,43 & 0,59 \\
\hline 3 & 1 & 16 & 36 & 9 & 1 & & & & & 1,89 & 0,72 \\
\hline 4 & & & 11 & 45 & 6 & 1 & & & & 2,95 & 0,58 \\
\hline 5 & & 2 & 24 & 33 & 4 & & & & & 2,62 & 0,66 \\
\hline 6 & & & 1 & 2 & 9 & 19 & 24 & 5 & 3 & 5,43 & 1,17 \\
\hline
\end{tabular}
quent dans l'échantillon a 3 racines sur $E_{2}, 2$ sur $E_{3}$ et 3 sur $E_{4}$ (Fig. 2).

Tableau I. Distribution des pieds, en fonction du nombre de racines par entrenœud. 
Tableau II. Matrice des corrélations entre nombres de racines sur les entrenœuds successifs.

\begin{tabular}{rrrrrr}
\hline & E2 & E3 & E4 & E5 & E6 \\
\hline E2 & 1 & & & & \\
E3 & $-0,364$ & 1 & & & \\
E4 & 0,149 & $-0,244$ & 1 & & \\
E5 & $-0,228$ & 0,317 & $-0,471$ & 1 & \\
E6 & 0,010 & $-0,019$ & 0,315 & $-0,307$ & 1 \\
\hline
\end{tabular}

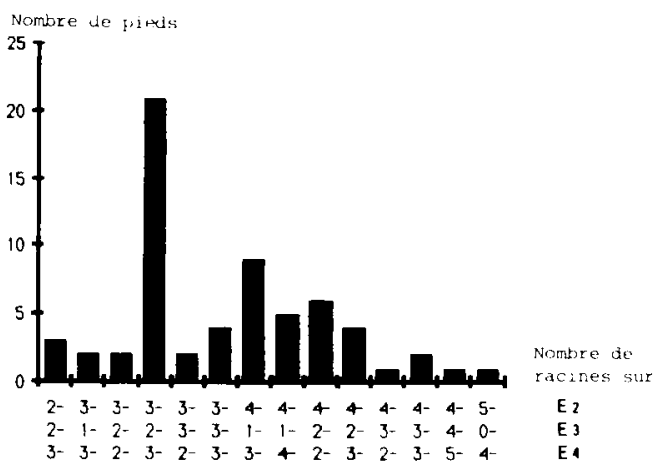

Fig. 2. Fréquence des différents types de pied.

\section{Disposition des racines sur un entrenœud donné}

Le calcul du $\chi^{2}$ pour tester l'hypothèse d'une disposition uniformément aléatoire et indépendante a été fait dans les cas suivants : entrenœud 2 portant 3 racines; entrenœud 3 portant 2 racines; entrenœud 4 portant 3 racines; entrenœud 5 portant 2 racines et entrenœud 5 portant 3 racines. Dans les autres cas, l'effectif était insuffisant. Dans tous les cas où le calcul a été fait, le test de $\chi^{2}$ conduit à rejeter l'hypothèse d'une disposition aléatoire et indépendante des racines (Tableau III).

Dans la Figure 3, on a représenté la distribution des valeurs du plus grand angle $\left(\lambda_{1}\right)$ pour les entrenœuds portant 2 racines. II apparaît très peu de valeurs à 180 degrés. Cela contredit l'hy- pothèse d'une disposition symétrique. On observe par contre un mode à 240 degrés.

Dans la Figure 4, on a représenté les valeurs relatives des 2 plus grands angles $\lambda_{1}$ et $\lambda_{2}$ pour les cas à 3 racines. L'aire comprise entre les 3 droites est la zone des valeurs possibles. II n'y a pas dispersion des points sur toute cette surface. On observe un grand nombre de points proches

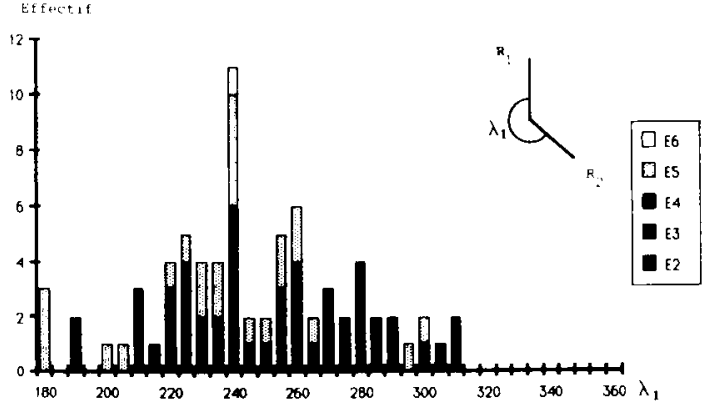

Fig. 3. Cas des entrenœuds ayant 2 racines. Distribution des valeurs du plus grand des 2 angles.

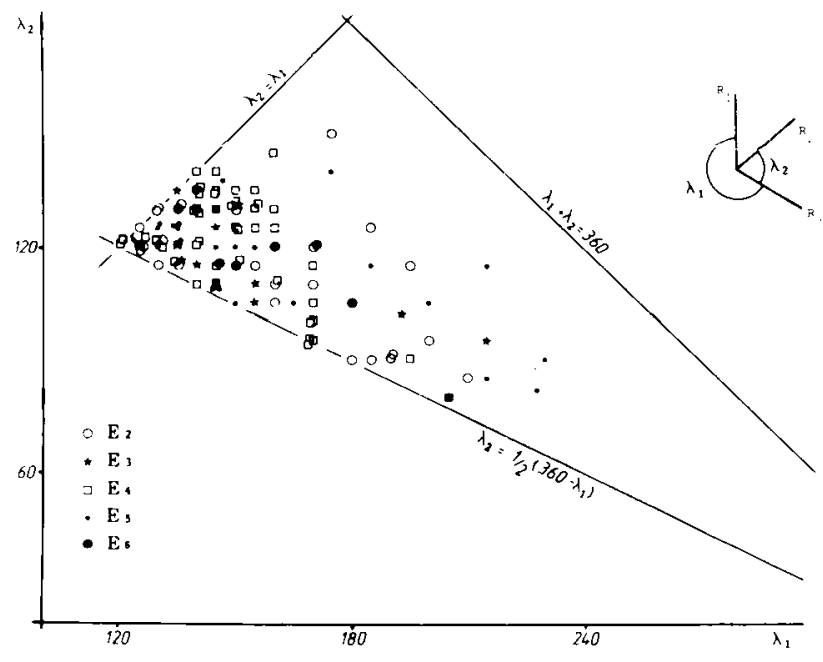

Fig. 4. Cas des entrenœuds ayant 3 racines. Valeurs relatives des 2 plus grands angles.

Tableau III. Comparaison des dispositions observées à un modèle uniformément aléatoire - Résultats du test de $\chi^{2}$.

\begin{tabular}{|c|c|c|c|c|c|}
\hline Entrenœuds & $\begin{array}{c}\text { Nombre de } \\
\text { racines }\end{array}$ & $\begin{array}{c}\text { Nombre de } \\
\text { pieds (n) } \\
\text { concernés }\end{array}$ & $\begin{array}{c}\text { Nombre de } \\
\text { classes }\end{array}$ & $d d l$ & $\chi^{2}$ obs \\
\hline
\end{tabular}

\begin{tabular}{rrrrrrr}
2 & 3 & 31 & 25 & 24 & 142,39 & 43,0 \\
3 & 2 & 36 & 5 & 30,94 & 13,3 \\
4 & 3 & 45 & 25 & 4 & 176,67 & 43,0 \\
5 & 24 & 5 & 24 & 16,83 & 13,3 \\
5 & 2 & 33 & 25 & 104,12 & 43,0 \\
\hline
\end{tabular}


du point ayant pour coordonnées $(120,120)$. La distribution de l'ensemble des valeurs d'angle entre racines présente un mode à 120 degrés (Fig. 5). On est proche dans ce cas d'une disposition symétrique.

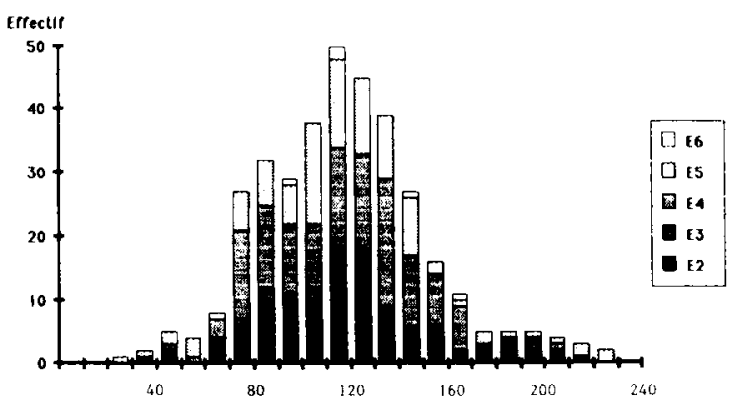

Fig. 5. Cas des entrenœuds ayant trois racines. Distribution des valeurs d'angle entre racines.

Dans les Figures $6 a$, b et $c$, on a représenté I'histogramme des valeurs d'angle entre racines pour les cas à 4,5 et 6 racines, respectivement. Sur l'axe des abscisses, on a fait apparaître la valeur de l'angle correspondant à une disposition symétrique: 90 degrés pour 4 racines, 72 degrés pour 5 racines, 60 degrés pour 6 racines. Dans les 3 cas, on observe une forte dispersion des valeurs. Le mode ne correspond pas à ce que donnerait une disposition symétrique, en particulier dans le cas à 4 racines où l'histogramme est bimodal. L'ensemble de ces observations conduit à rejeter l'hypothèse d'une disposition symétrique. Cette hypothèse est démentie pour $\mathrm{I}=2$ et $\mathrm{I}=4$.

\section{Disposition des racines sur 2 entrencuds successifs}

\section{Angles occupés}

Dans le Tableau IV , on a considéré l'ensemble des angles entre racines sur $E_{i}(i=1$ à 5$)$ et on a calculé par classe de valeurs la fréquence des cas où ces angles contiennent 0,1 ou plusieurs racines. Les limites des classes ont été choisies de telle sorte que les effectifs par classe soient proches. Dans $79,8 \%$ des cas, les angles inférieurs à 80 degrés ne contiennent aucune racine. Les angles de 0 à 100 degrés ne contiennent jamais plus de 2 racines, les angles de 100 à 140 jamais plus de 3 et les angles de 140 à 200 jamais plus de 4 . Dans la Figure 7 , on a fait apparaître, en fonction de la valeur de l'angle sur $E_{i}$, le nombre moyen de racines de $E_{i+1}$ contenues et le rapport nombre de racines contenues sur valeur de l'angle. Ce rapport mesure le nombre de racines contenues par unité d'angle. II est constant pour les angles supérieurs à 100
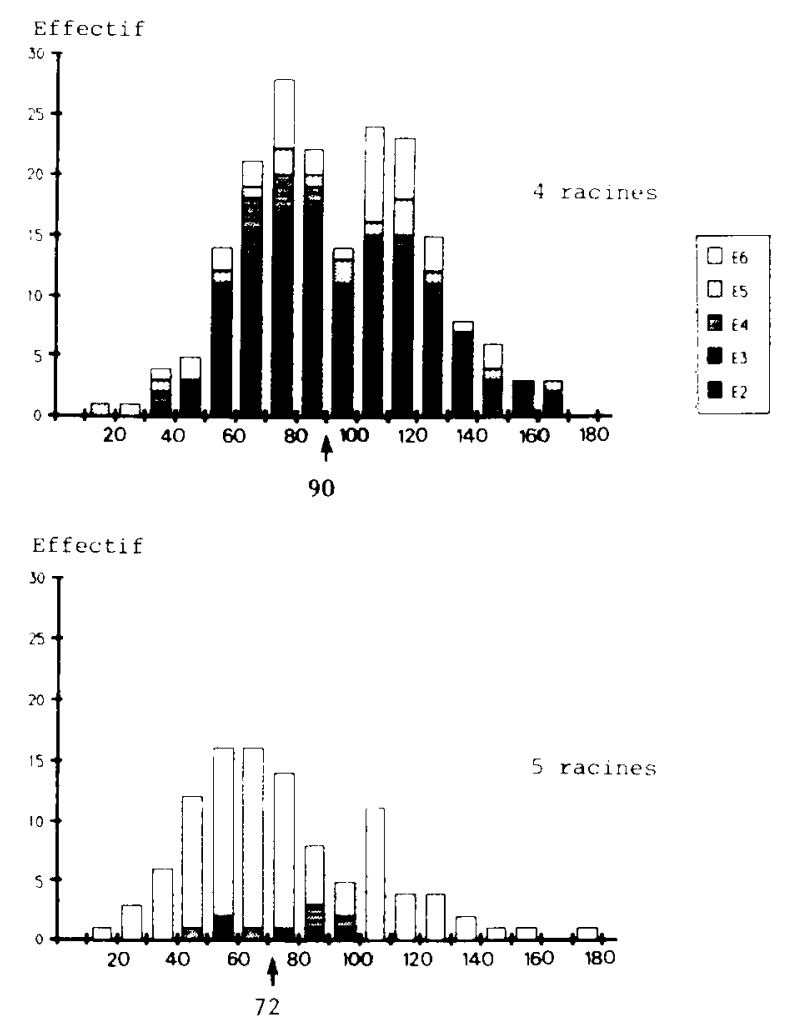

Effectif

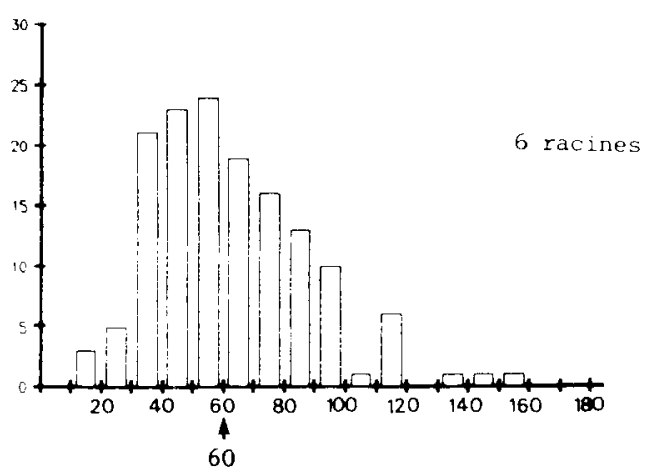

Fig. 6. Distribution des valeurs d'angle entre racines : a entrenœuds portant 4 racines; $b$ - entrenœuds portant 5 racines; $c-$ entrenœuds portant 6 racines.

degrés. Par contre, il est plus faible pour les angles inférieurs à 100 degrés.

Dans l'échantillon, nous avons recensé 29 combinaisons de valeurs $\left(l_{i}, l_{i+1}\right)$. La position des racines de $E_{i+1}$ par rapport aux angles entre racines sur $E_{i}$ classés par ordre décroissant a été étudiée pour les combinaisons les plus représentées. Pour chaque combinaison, on a noté entre parenthèses le pourcentage qu'elle représente dans l'échantillon. Les résultats sont les suivants: - cas 1: 4 racines sur $E_{i}, 1$ sur $E_{i+1}(6,35 \%)$. La racine de $E_{i+1}$ occupe l'angle le plus ouvert sur $\mathrm{E}_{i}\left(\lambda_{1}\right)$ dans $87,5 \%$ des cas.

- cas $2: 3$ racines sur $E_{i}, 2$ sur $E_{i+1}(18,25 \%)$. Dans $67,4 \%$ des cas, les 2 racines de $E_{i+1}$ occupent respectivement les 2 angles les plus ouverts sur $E_{i}\left(\lambda_{1}\right.$ et $\left.\lambda_{2}\right)$. 
Tableau IV. Fréquence des cas où un angle entre racines sur $E_{i}$ contient 0,1 ou plusieurs racines de $E_{i+1}$, pour chaque classe d'angle entre racines sur $E_{j}$.

\begin{tabular}{|c|c|c|c|c|c|c|c|c|c|c|c|}
\hline \multirow{2}{*}{$\begin{array}{l}\text { Classe } \\
\text { d'angle sur } E_{i} \\
\text { (en degrés) }\end{array}$} & \multirow{2}{*}{$\begin{array}{c}\text { Valeur } \\
\text { moyenne } \\
\text { (en degrés) }\end{array}$} & \multirow{2}{*}{ Effectif } & \multirow[b]{2}{*}{0} & \multirow[b]{2}{*}{1} & \multirow[b]{2}{*}{2} & \multicolumn{3}{|c|}{ Nombre de racines de $E_{i+1}$} & \multirow[b]{2}{*}{6} & \multirow[b]{2}{*}{7} & \multirow[b]{2}{*}{8} \\
\hline & & & & & & 3 & 4 & 5 & & & \\
\hline 0,80 & 60,89 & 104 & 0,798 & 0,173 & 0,029 & 0 & 0 & 0 & 0 & 0 & 0 \\
\hline 80,100 & 87,10 & 115 & 0,548 & 0,409 & 0,043 & 0 & 0 & 0 & 0 & 0 & 0 \\
\hline 100,120 & 107,72 & 120 & 0,200 & 0,600 & 0,183 & 0,017 & 0 & 0 & 0 & 0 & 0 \\
\hline $120, \quad 140$ & 126,86 & 137 & 0,066 & 0,708 & 0,212 & 0,015 & 0 & 0 & 0 & 0 & 0 \\
\hline 140,200 & 157,79 & 114 & 0,018 & 0,605 & 0,272 & 0,061 & 0,044 & 0 & 0 & 0 & 0 \\
\hline $200, \quad 360$ & 267,40 & 96 & 0 & 0,021 & 0,510 & 0,219 & 0,188 & 0,010 & 0,042 & 0 & 0,010 \\
\hline Total & & 686 & 0,264 & 0,445 & 0,203 & 0,047 & 0,034 & 0,001 & 0,006 & 0 & 0,001 \\
\hline
\end{tabular}

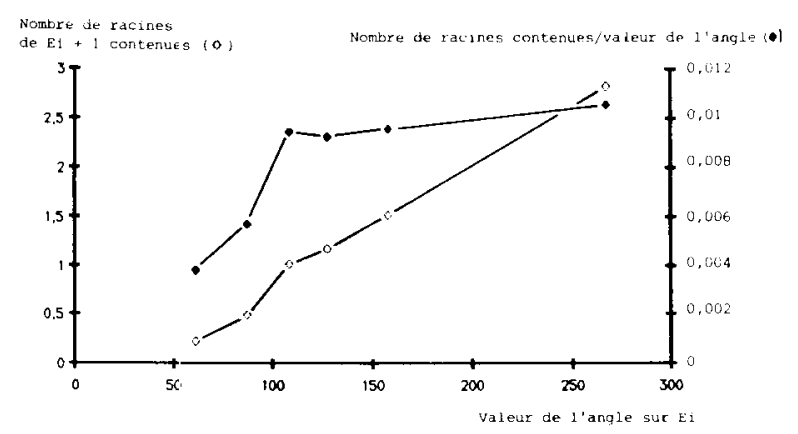

Fig. 7. Nombre de racines de $E_{i+1}$ contenues entre 2 racines de $E_{i}$, rapporté à la valeur de l'angle entre les 2 racines de $E_{i}$.

- cas $3: 3$ racines sur $E_{i}, 3$ sur $E_{i+1}(15,08 \%)$. Les 3 racines de $E_{i+1}$ occupent respectivement les 3 angles sur $E_{i}$ dans $73,7 \%$ des cas. Dans $21,1 \%$ des cas, on trouve 2 racines dans $\lambda_{1}$ et une dans $\lambda_{2}$.

- cas $4: 2$ racines sur $E_{i}, 3$ sur $E_{i+1}(14,29 \%)$. Dans $88,9 \%$ des cas, 2 racines se trouvent dans $\lambda_{1}$ et une dans $\lambda_{2}$.

Dans le Tableau $V$, on a calculé dans le cas 1 la valeur moyenne de chacun des angles $\lambda_{1}, \lambda_{2}$, $\lambda_{3}$ et $\lambda_{4}$. Si la position angulaire de la racine de $E_{i+1}$ était aléatoire dans l'intervalle [0,360[, la probabilité qu'elle apparaisse dans chacun des angles $\lambda_{1}, \lambda_{2}, \lambda_{3}$ et $\lambda_{4}$ serait proportionnelle à la valeur de l'angle. Nous avons calculé sous cette hypothèse la fréquence théorique d'apparition de la racine de $E_{i+1}$ dans chacun de ces angles. La fréquence observée d'apparition de la racine de $E_{i+1}$ dans l'angle le plus ouvert $\left(\lambda_{1}\right)$ est supérieure à la fréquence calculée. L'ensemble de ces observations montrent que les racines de $E_{i+1}$ occupent en priorité les angles les plus ouverts sur $\mathrm{E}_{i \text {. }}$
Tableau V. Position de la racine de l'entrenœud $E_{i+1}$ relativement aux angles entre racines sur l'entrenœud $E_{i}$, classés par ordre décroissant $\left(\lambda_{1}>\lambda_{2}>\lambda_{3}>\lambda_{4}\right)$, dans le cas où il y a 1 racine sur $E_{i+1}$ et 4 sur $E_{i}$. $\lambda_{i}$ - valeur moyenne de l'angle dans l'échantillon. $F$ théorique = fréquence calculée d'apparition de la racine de $E_{i+1}$ dans chacun des angles $\lambda_{i}$ si on suppose sa position aléatoire. $F$ observée = fréquence observée d'apparition de la racine de $E_{i+1}$ dans chacun des angles $\lambda_{i}$.

\begin{tabular}{lrrrr}
\hline & \multicolumn{1}{c}{$\lambda_{1}$} & \multicolumn{1}{c}{$\lambda_{2}$} & \multicolumn{1}{c}{$\lambda_{3}$} & \multicolumn{1}{c}{$\lambda_{4}$} \\
\hline$\lambda_{i}$ & 126,6 & 96,6 & 77,2 & 59,7 \\
F théorique & 35,2 & 26,8 & 21,4 & 16,6 \\
F observée & 87,5 & 6,2 & 6,2 & 0,0 \\
\hline
\end{tabular}

\section{Position des racines de $E_{i+1}$ dans les angles occupés}

Dans $44,5 \%$ des cas, un angle entre 2 racines sur $E_{i}$ est occupé par une seule racine de $E_{i+1}$. Dans la Figure 8, on a calculé l'angle entre cette racine et la bissectrice (noté $\alpha$ ) rapporté à la valeur du demi-angle (noté $\beta$ ). La distribution obtenue montre que dans la majorité des cas, la racine de $E_{i+1}$ a une position proche de la bissectrice.

Le même calcul a été fait dans le cas où un angle sur $E_{i}$ est occupé par 2 racines de $E_{i+1}$. Les écarts à la bissectrice de ces 2 racines sont presque toujours de signe opposé, ce qui veut dire qu'elles se situent de part et d'autre de la bissectrice. Si les 2 racines de $E_{i+1}$ découpaient l'angle qu'elles occupent en 3 angles égaux, les valeurs calculées seraient proches respectivement de $-1 / 3$ et $+1 / 3$. On observe de fait des 
modes proches de ces valeurs, mais légèrement décalés (inférieurs à $-1 / 3$ et supérieurs à $+1 / 3$ ) (Fig. 9 a et b). Les valeurs moyennes obtenues sont respectivement $-0,405$ et $+0,390$. Cela signifie que les 2 racines de $E_{i+1}$ s'écartent plus l'une de l'autre qu'elles ne s'écartent des racines de l'entrenœud précédent.

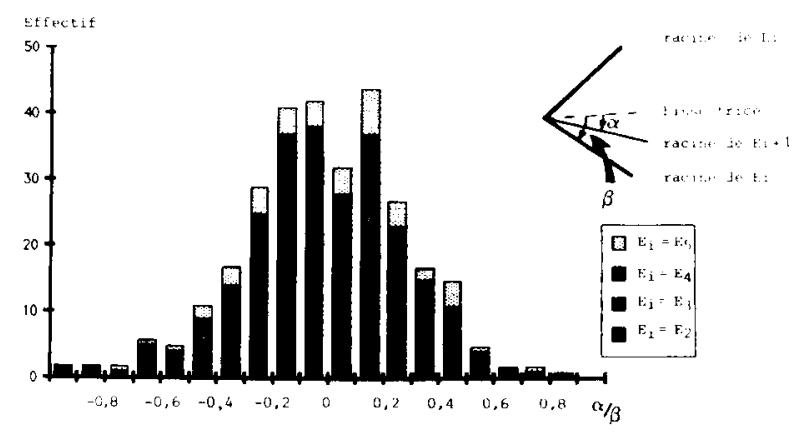

Fig. 8. Cas où un angle entre 2 racines sur $E_{i}$ est occupé par une seule racine $E_{i+1}$. Position de cette racine par rapport à la bissectrice.
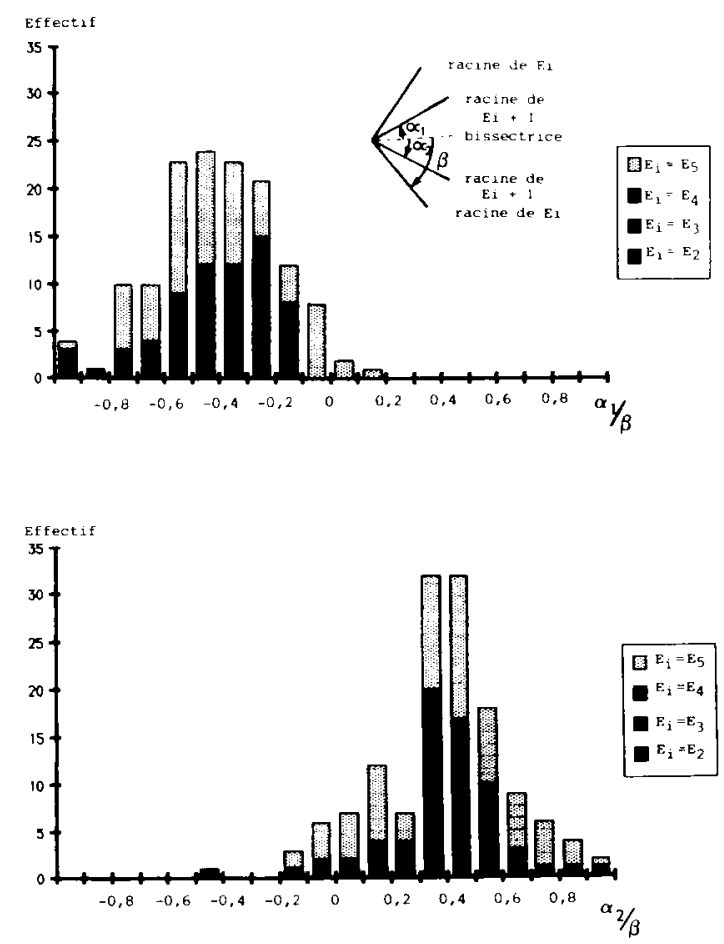

Fig. 9. Cas où un angle entre 2 racines sur $E_{i}$ est occupé par 2 racines de $E_{i+1}$ a - Position de la première racine (dans $l e$ sens trigonométrique); $b$ - Position de la deuxième racine.

\section{DISCUSSION - CONCLUSION}

L'analyse qui précède a montré que sur un entrenœud donné la disposition des racines n'est ni aléatoire ni symétrique. Sur 2 entrenœuds successifs, les dispositions sont alternées. Les racines d'un entrenœud occupent en priorité les angles les plus ouverts sur l'entrenœud précédent. Une racine comprise entre 2 racines de l'entrenœud précédent a une position proche de la bissectrice. Cela permet d'expliquer certains résultats obtenus en considérant les entrenœuds un par un. Dans le cas d'un entrenœud portant 2 racines, nous avons observé un grand nombre de dispositions à 120 degrés. Cela peut résulter de la disposition des racines entre les racines de l'entrenœud précédent si celui-ci comporte 3 racines.

L'analyse est cependant incomplète, car on se heurte rapidement à des difficultés de représentation des données. La description simple des données ne permet pas d'étudier complètement les interactions entre nombre de racines, disposition sur l'entrenœud, nombre et disposition sur l'entrenœud précédent. L'analyse n'a pas non plus pris en compte l'évolution des diamètres de la tige et des racines sur les entrenœuds successifs. Enfin, nous n'avons pas tenu compte d'éventuelles dispositions privilégiées des racines par rapport aux organes aériens proches, tels que les bourgeons axillaires.

L'existence d'une rhizotaxie renvoie au processus d'ontogénèse des racines adventives. Chez le maïs, celle-ci a déjà été décrite (Hoppe et al., 1986; Girardin et al., 1987). Les ébauches se forment à partir de massifs cellulaires résultant de la dédifférenciation de cellules parenchymateuses situées à la périphérie de la tige. Leur origine est donc superficielle. Sur les entrenœuds $E_{2}$ et $E_{5}$, la totalité des ébauches présentes s'allongent. Au-delà de $E_{6}$, il y a moins de racines allongées que d'ébauches différenciées (Girardin et al. 1987). Les coupes histologiques réalisées à l'occasion de ces travaux n'ont pas permis de mettre en évidence une situation privilégiée des ébauches par rapport aux structures internes de la tige. Les faisceaux conducteurs à la périphérie de la tige sont plus nombreux que les ébauches racinaires formées. D'après Hoppe et al. (1986), il y a formation d'un anneau prévasculaire passant à la base des primordia racinaires après apparition de ceux-ci. Cet anneau forme ensuite des cônes qui pénètrent les primordia racinaires et assurent ainsi les connexions racine-tige. La position des racines adventives ne serait donc pas prédéterminée par la structure vasculaire de la tige.

Pour la construction d'un modèle d'architecture du système racinaire du maïs, on peut retenir les dispositions les plus fréquentes ou bien tenter une modélisation probabiliste. Les travaux et modèles existants traitant du positionnement des organes chez les végétaux concernent surtout la disposition des feuilles sur la tige. Plusieurs hypothèses ont été avancées pour expliquer l'existence de dispositions particulières. D'après Snow (1962, cité par Jean, 1983), les primordia 
se forment dans les espaces entre 2 primordia existants, dès que l'un de ces espaces a atteint une taille suffisante. D'après Richards (19481951, cité par Jean, 1983) les primordia sont entourés d'une $z^{\urcorner}$ne d'inhibition d'origine hormonale empêchant la formation d'autres primordia dans leur voisinage immédiat. Cela explique par exemple que les nouveaux primordia apparaissent dans l'espace le plus ouvert entre 2 primordia existants. Ce type d'hypothèse peut rendre compte des dispositions observées des racines autour de la tige. Un modèle a été développé et sera présenté dans un deuxième article.

\section{RÉFÉRENCES}

Diggle A.J. (1988) Rootmap - a model in three - dimensional coordinates of the growth and structure of fibrous root systems. Plant Soil 105, 169-178

Girardin P., Jordan M-O., Picard D. \& Trendel R. (1986) Harmonisation des notations concernant la description morphologique d'un pied de maïs (Zea mays L.), Agronomie 6, 873-875

Girardin P., Morel-Fourrier B., Jordan M-O. \& Millet B. (1987) Développement des racines adventives chez le maïs. Agronomie 7, 353-360

Hoppe D.C., Mc Cully M.E. \& Wenzel C-L. (1986) The nodal roots of Zea : their development in relation to structural features of the stem. Can. J. Bot 64, 2 5242537

Jean R.V. (1983) Mathematical modeling in phyllotaxis $=$ the stage of the art. Math. Bio. Sci 64, 1-27

Jordan M-O., Girardin P., Varlet-Grancher C., Picard D. \& Trendel R. (1988) Rythme d'apparition des racines primaires du maïs (Zea mays L.). III. Variations observées au champ. Agronomie 8, 37-46

Klepper B., Belford R.K. \& Rickman R.W. (1984) Root and shoot development in winter wheat. Agron. J. 76, 117-122

Lungley D.R. (1973) The growth of root systems. A numerical computer simulation model. Plant Soil 38 , 145-159

Pages L. \& Aries F. (1988) Sarah : modèle de simulation de la croissance, du développement et de l'architecture des systèmes racinaires. Agronomie 8, 889896

Picard D., Jordan M-O. \& Trendel R. (1985) Rythme d'apparition des racines primaires du maïs (Zea mays L.). I. Etude détaillée pour une variété en un lieu donné. Agronomie 5, 667-676

Porter J.R., Klepper B. \& Belford R.K. (1986) A model (WHTROOT) which synchronizes root growth and development with shoot development for winter wheat. Plant Soil 92, 133-145

Tardieu F. (1987) Etat structural, enracinement et alimentation hydrique du maïs. III. Disponibilité des réserves en eau du sol. Agronomie 7, 279-288 